\title{
Gupta-Bleuler quantization for massive and massless free vector fields
}

\author{
M. Dehghani* \\ Department of Physics, Ilam University, Ilam, Iran
}

(Received on 28 April, 2009)

\begin{abstract}
It is shown that the usual quantum field theory leads to an ultraviolet divergence in the vacuum energies and an infrared divergence in the two-point functions of the massive and massless vector fields. Using a new method of quantization it is shown that the vacuum energies converge, and the normal ordering procedure is not necessary. Also the propagators are calculated, which are automatically renormalied.

Keywords: vector fields, quantization, Gupta-Bleuler
\end{abstract}

\section{INTRODUCTION}

Particles of spin-1 are described as the quanta of vector fields. Such vector bosons play a central role as the mediators of interactions in particle physics. The important examples are the gauge fields of the electromagnetic (massless photons), the weak (massive $W^{ \pm}$and $Z^{0}$ bosons), and the strong (massless gluons) interactions. On a somewhat less fundamental level vector fields can also be used to describe spin- 1 mesons, for example the $\rho$ and the $\omega$ mesons.

It has been shown $[1,2]$ that one can construct a covariant quantization of massless minimally coupled scalar field in de Sitter space-time, which is causal and free of any divergences. The essential point of these papers is the unavoidable presence of the negative norm states. Also they do not propagate in the physical space, they play a renormalization role. In the previous paper [3] we have shown that this is also true for linear gravity (the traceless rank-2 massless tensor field). These questions have recently been studied by several authors [4-7].

These auxiliary states (the negative norm states) appear to be necessary for obtaining a fully covariant quantization of the free minimally coupled scalar field in de Sitter spacetime, which is free of any infrared divergence. It has been shown that these auxiliary states automatically renormalize the infrared divergence in the two-point function [3, 4] and remove the ultraviolet divergence in the vacuum energy [2]. This method is applied to the massless vector field in de Sitter space $[8,9]$, interacting quantum fields and Casimir effect in Mincowski space-time [10-12].

We would like to generalize this quantization method to the massive and massless free vector fields in Minkowski space-time. These auxiliary states once again, automatically leads to renormalized two-point functions and removes the ultraviolet divergences in the vacuum energies.

This new quantization method is used for consideration of the Casimir effect for the scalar field [10]. It can be extended for the electromagnetic field in 4-dimensional spacetime. The crucial point is that when we impose the physical boundary conditions on the field operator, only the positive norm states are affected. The negative norm states do not interact with the physical states or real physical world, thus they cannot be affected by the physical boundary conditions

*e-mail:dehghan22@gmail.com as well. The results for the Casimir energy and Casimir force are the same given in $[13,14]$.

In section 2 a brief review of the massive vector field quantization, using usual quantum field theory is presented. The ultraviolet divergence of the vacuum energy and the infrared divergence of the two-point function is explicitly shown for this field. Then using Gupta-Bleuler quantization it is shown that the vacuum energy converges and the infrared divergence of the propagator disappears. In section 3 the ultraviolet divergence of the vacuum energy and the infrared divergence of the two-point function of the massless vector field is shown clearly in the framework of usual quantum field theory. Also the vacuum energy and the propagator of this field is calculated using Gupta-Bleuler quantization, which are free of any divergences. Finally a brief conclusion is given in section 4 .

\section{MASSIVE VECTOR FIELD}

The lagrangian density for the massive vector field $A_{\mu}(x)$, in Minkowskian flat space-time is that of Proca [13]

$$
\mathcal{L}=-\frac{1}{4} F_{\mu v} F^{\mu \nu}+\frac{1}{2} m^{2} A_{\mu} A^{\mu}
$$

where $F_{\mu v}=\partial_{\mu} A_{v}-\partial_{v} A_{\mu}$. The variational principal to (2.1) yields the field equation

$$
\partial^{\mu} F_{\mu v}+m^{2} A_{v}=0 .
$$

Taking the divergence of Eq. (2.2) we have $m^{2} \partial^{v} A_{v}=0$, and since $m^{2} \neq 0$, we find the Lorenz gauge and Eq.(2.2) can be written as

$$
\square A_{\mu}+m^{2} A_{\mu}=0, \quad \partial_{\mu} A^{\mu}=0 .
$$

\subsection{Usual quantum field theory}

There are three independent components of a massive spin-1 field, and the Lorentz condition may serve to eliminate one of the four components of $A_{\mu}$; we take this to be $A^{0}$. Note that the positive sign in front of the mass term in the Lagrangian will result in a term $-\frac{1}{2} m^{2}$ (A.A) when $A^{0}$ is eliminated, so that the mass term has a negative coefficient, as it does for the Klein-Gordon Lagrangian. The field $A_{\mu}(x)$ 
has the expansion

$A_{\mu}(x)=\int \frac{d^{3} \mathbf{k}}{(2 \pi)^{3} 2 \omega_{k}} \sum_{\lambda=1}^{3} \varepsilon_{\mu}^{(\lambda)}(k)\left[a^{(\lambda)}(\mathbf{k}) e^{-i k . x}+a^{(\lambda) \dagger}(\mathbf{k}) e^{i k . x}\right]$,

in which $\omega_{k}=\sqrt{\mathbf{k}^{2}+m^{2}}$, and

$$
\begin{gathered}
{\left[a^{(\lambda)}(\mathbf{k}), a^{\left(\lambda^{\prime}\right) \dagger}\left(\mathbf{k}^{\prime}\right)\right]=2 \omega_{k}(2 \pi)^{3} \delta_{\lambda \lambda^{\prime}} \delta^{3}\left(\mathbf{k}-\mathbf{k}^{\prime}\right),} \\
{\left[a^{(\lambda)}(\mathbf{k}), a^{\left(\lambda^{\prime}\right)}\left(\mathbf{k}^{\prime}\right)\right]=\left[a^{(\lambda) \dagger}(\mathbf{k}), a^{\left(\lambda^{\prime}\right) \dagger}\left(\mathbf{k}^{\prime}\right)\right]=0 .}
\end{gathered}
$$
tions,

The polarization vectors $\varepsilon_{\mu}^{(\lambda)}(k)$, obey the following rela-

$$
\varepsilon^{(\lambda)} \cdot \varepsilon^{\left(\lambda^{\prime}\right)}=\delta_{\lambda \lambda^{\prime}}, \quad \varepsilon_{\mu}^{(\lambda)}(k) k^{\mu}=0
$$

It may be checked out that the Hamiltonian is

$H=\sum_{\lambda} \int \frac{d^{3} \mathbf{k}}{(2 \pi)^{3} 2 k_{0}} \frac{k_{0}}{2}\left[a^{(\lambda)}(\mathbf{k}) a^{(\lambda) \dagger}(\mathbf{k})+a^{(\lambda) \dagger}(\mathbf{k}) a^{(\lambda)}(\mathbf{k})\right]$.

It is clear that the above integral and so the vacuum energy diverges. Unless the "normal ordering" procedure is used.

We now calculate the propagator for massive spin- 1 particles. Recall that the relation between the propagator and the two-point function is [13]

$$
\left\langle 0\left|T\left(A_{\mu}(x) A_{v}(y)\right)\right| 0\right\rangle=i G_{\mu v}(x-y) .
$$

We calculate the left-hand side by substituting $A_{\mu}(x)$ from (2.4)

$$
\left\langle 0\left|T\left(A_{\mu}(x) A_{v}(y)\right)\right| 0\right\rangle=i \int \frac{d^{4} k}{(2 \pi)^{4}} \frac{e^{-i k .(x-y)}}{k^{2}-m^{2}+i \varepsilon} \sum_{\lambda=1}^{3} \varepsilon_{\mu}^{(\lambda)}(k) \varepsilon_{v}^{(\lambda)}(k)
$$

By a direct calculation one can show that this propagator bears an infrared divergence and the divergent term appears in it's imaginary part (Appendix-A).

\subsection{Gupta-Bleuler quantization}

In the Krein quantum field theory the field operator is defined as follows $[2,4,11]$

$$
\begin{gathered}
A_{\mu}(x)=\frac{1}{\sqrt{2}}\left[A_{\mu}^{(p)}(x)+A_{\mu}^{(n)}(x)\right] \\
A_{\mu}^{(p)}(x)=\int \frac{d^{3} \mathbf{k}}{(2 \pi)^{3} 2 \omega_{k}} \sum_{\lambda=1}^{3} \varepsilon_{\mu}^{(\lambda)}(k)\left[a^{(\lambda)}(\mathbf{k}) e^{-i k \cdot x}\right. \\
\left.+a^{(\lambda) \dagger}(\mathbf{k}) e^{i k . x}\right] \\
A_{\mu}^{(n)}(x)=\int \frac{d^{3} \mathbf{k}}{(2 \pi)^{3} 2 \omega_{k}} \sum_{\lambda=1}^{3} \varepsilon_{\mu}^{(\lambda)}(k)\left[b^{(\lambda)}(\mathbf{k}) e^{i k . x}\right. \\
\left.+b^{(\lambda) \dagger}(\mathbf{k}) e^{-i k \cdot x}\right]
\end{gathered}
$$

where $A_{\mu}^{(p)}(x)$ and $A_{\mu}^{(n)}(x)$ have positive and negative norm respectively, in the sense of Klein-Gordon inner product. Creation and annihilation operators obey the following commutation relations

$$
\begin{aligned}
{\left[a^{(\lambda)}(\mathbf{k}), a^{\left(\lambda^{\prime}\right)}\left(\mathbf{k}^{\prime}\right)\right] } & =0,\left[a^{(\lambda) \dagger}(\mathbf{k}), a^{\left(\lambda^{\prime}\right) \dagger}\left(\mathbf{k}^{\prime}\right)\right]=0, \\
{\left[a^{(\lambda)}(\mathbf{k}), a^{\left(\lambda^{\prime}\right) \dagger}\left(\mathbf{k}^{\prime}\right)\right] } & =2 \omega_{k}(2 \pi)^{3} \delta_{\lambda \lambda^{\prime}} \delta^{3}\left(\mathbf{k}-\mathbf{k}^{\prime}\right), \\
{\left[b^{(\lambda)}(\mathbf{k}), b^{\left(\lambda^{\prime}\right)}\left(\mathbf{k}^{\prime}\right)\right] } & =0,\left[b^{(\lambda) \dagger}(\mathbf{k}), b^{\left(\lambda^{\prime}\right) \dagger}\left(\mathbf{k}^{\prime}\right)\right]=0,
\end{aligned}
$$

$$
\begin{array}{r}
{\left[b^{(\lambda)}(\mathbf{k}), b^{\left(\lambda^{\prime}\right) \dagger}\left(\mathbf{k}^{\prime}\right)\right]=-2 \omega_{k}(2 \pi)^{3} \delta_{\lambda \lambda^{\prime}} \delta^{3}\left(\mathbf{k}-\mathbf{k}^{\prime}\right),} \\
{\left[a^{(\lambda)}(\mathbf{k}), b^{(\lambda)}\left(\mathbf{k}^{\prime}\right)\right]=0, \quad\left[a^{(\lambda) \dagger}(\mathbf{k}), b^{\left(\lambda^{\prime}\right) \dagger}\left(\mathbf{k}^{\prime}\right)\right]=0,} \\
{\left[a^{(\lambda)}(\mathbf{k}), b^{\left(\lambda^{\prime}\right) \dagger}\left(\mathbf{k}^{\prime}\right)\right]=0, \quad\left[a^{(\lambda) \dagger}(\mathbf{k}), b^{\left(\lambda^{\prime}\right)}\left(\mathbf{k}^{\prime}\right)\right]=0 .}
\end{array}
$$

The vacuum state $|0\rangle$ is then defined by

$$
\begin{gathered}
a^{(\lambda) \dagger}(\mathbf{k})|0\rangle=\left|1_{\mathbf{k}}\right\rangle, \quad a^{(\lambda)}(\mathbf{k})|0\rangle=0, \quad \forall \mathbf{k}, \lambda, \\
b^{(\lambda) \dagger}(\mathbf{k})|0\rangle=\left|\overline{1}_{\mathbf{k}}\right\rangle, \quad b^{(\lambda)}(\mathbf{k})|0\rangle=0, \quad \forall \mathbf{k}, \lambda, \\
b^{(\lambda)}(\mathbf{k})\left|1_{\mathbf{k}}\right\rangle=0, \quad a^{(\lambda)}(\mathbf{k})\left|\overline{1}_{\mathbf{k}}\right\rangle=0, \quad \forall \mathbf{k}, \lambda,
\end{gathered}
$$

where $\left|1_{\mathbf{k}}\right\rangle$ is called a one particle state and $\left|\overline{1}_{\mathbf{k}}\right\rangle$ is called a one "unparticle state". These commutation relations, together with the normalization of the vacuum

$$
\langle 0 \mid 0\rangle=1
$$

lead to positive (negative) norms on the physical (unphysical) sector:

$$
\left\langle 1_{\mathbf{k}} \mid 1_{\mathbf{k}^{\prime}}\right\rangle=\delta^{3}\left(\mathbf{k}-\mathbf{k}^{\prime}\right), \quad\left\langle\overline{1}_{\mathbf{k}} \mid \overline{1}_{\mathbf{k}^{\prime}}\right\rangle=-\delta^{3}\left(\mathbf{k}-\mathbf{k}^{\prime}\right) .
$$

Using the field operator (2.10) one can show that

$$
\begin{aligned}
& H=\sum_{\lambda=1}^{3} \int \frac{d^{3} \mathbf{k}}{(2 \pi)^{3} 2 k_{0}} \frac{k_{0}}{2}\left[a^{(\lambda) \dagger}(\mathbf{k}) a^{(\lambda)}(\mathbf{k})\right. \\
& \left.+b^{(\lambda) \dagger}(\mathbf{k}) b^{(\lambda)}(\mathbf{k})+a^{(\lambda) \dagger}(\mathbf{k}) b^{(\lambda) \dagger}(\mathbf{k})+a^{(\lambda)}(\mathbf{k}) b^{(\lambda)}(\mathbf{k})\right] .
\end{aligned}
$$


This energy is zero for the vacuum state and it is not needed to use the "normal ordering" procedure. It is also positive for any particles state or physical state $\left|N_{\mathbf{k}}\right\rangle$ (those built from repeated action of the $a^{\dagger}(\mathbf{k})$ 's on the vacuum)

$$
\begin{aligned}
& \left\langle N_{\mathbf{k}}|H| N_{\mathbf{k}}\right\rangle=\sum_{\lambda=1}^{3} \int \frac{d^{3} \mathbf{k}}{(2 \pi)^{3} 2 k_{0}} \frac{k_{0}}{2} \\
& \times\left\langle N_{\mathbf{k}}\left|a^{(\lambda) \dagger}(\mathbf{k}) a^{(\lambda)}(\mathbf{k})\right| N_{\mathbf{k}}\right\rangle \geq 0, \quad\left|0_{\mathbf{k}}\right\rangle \equiv|0\rangle .
\end{aligned}
$$

At this stage we calculate the propagator for the massive spin-1 particles, using Gupta-Bleuler quantization. Using (2.10) in the left hand side of (2.8) we have

$$
\begin{gathered}
\left\langle 0\left|T\left(A_{\mu}(x) A_{v}(y)\right)\right| 0\right\rangle=\frac{1}{2}\left[\left\langle 0\left|T\left(A_{\mu}^{(p)}(x) A_{v}^{(p)}(y)\right)\right| 0\right\rangle\right. \\
\left.+\left\langle 0\left|T\left(A_{\mu}^{(n)}(x) A_{\nu}^{(n)}(y)\right)\right| 0\right\rangle\right] \\
=\frac{1}{2}\left[W_{\mu \nu}^{(p)}(x, y)+W_{\mu \nu}^{(n)}(x, y)\right] .
\end{gathered}
$$

By a direct calculation one can show that $W_{\mu \nu}^{(n)}(x, y)=$ $-\left[W_{\mu \nu}^{(p)}(x, y)\right]^{*}$ and $W_{\mu \nu}^{(p)}(x, y)$ is the two-point function for the positive modes as it is calculated in the previous subsection. Therefore

$$
\begin{gathered}
\left\langle 0\left|T\left(A_{\mu}(x) A_{v}(y)\right)\right| 0\right\rangle=i I_{m}\left[W_{\mu \nu}^{(p)}(x, y)\right] \\
=i I_{m}\left[i \int \frac{d^{4} k}{(2 \pi)^{4}} \frac{e^{-i k \cdot(x-y)}}{k^{2}-m^{2}+i \varepsilon} \sum_{\lambda=1}^{3} \varepsilon_{\mu}^{(\lambda)}(k) \varepsilon_{v}^{(\lambda)}(k)\right],
\end{gathered}
$$

and the propagator for the transverse photon in the Krein space quantization is then

$G_{\mu v}(x-y)=i \mathcal{R} e\left[\int \frac{d^{4} k}{(2 \pi)^{4}} \frac{e^{-i k .(x-y)}}{k^{2}-m^{2}+i \varepsilon} \sum_{\lambda=1}^{3} \varepsilon_{\mu}^{(\lambda)}(k) \varepsilon_{v}^{(\lambda)}(k)\right]$

which is free of any infrared divergence and it is not need to use the renormalization procedure. Indeed this quantization method acts as an automatic renormalization factor for infrared divergence in the two-point function.

\section{MASSLESS VECTOR FIELD}

The six components of the electromagnetic field (three of electric and three of magnetic field) may be written as an antisymmetric tensor $F^{\mu v}$, and the homogeneous Maxwell equations follow if it is assumed that $F^{\mu v}$ is a 4-dimensional curl of the field $A_{\mu}$

$$
\begin{gathered}
F^{\mu v}=\partial^{\mu} A^{v}-\partial^{v} A^{\mu}, \\
\partial_{\mu} F^{\mu v}=0, \\
\square A^{v}-\partial^{v}\left(\partial_{\mu} A^{\mu}\right)=0 .
\end{gathered}
$$

These equations follow from a variational principle with the Lagrangian [13]

$$
\mathcal{L}=-\frac{1}{4} F_{\mu \nu} F^{\mu \nu}
$$

where $A_{\mu}$ is regarded as the dynamical field. The gauge transformation $A_{\mu} \rightarrow A_{\mu}^{\prime}=A_{\mu}+\partial_{\mu} \Lambda(x)$, leaves $F^{\mu \nu}$ unchanged. Potentials satisfying this additional condition,

$$
\phi=0, \quad \nabla . \mathbf{A}=0,
$$

are said to belong to the radiation (or Coulomb) gauge. In this gauge there are clearly only two independent components of $A_{\mu}$. This is the case in the real world, so working in the radiation gauge keeps the physical nature of the electromagnetic field most evident. Let us therefore study quantization in this gauge.

\subsection{Usual quantum field theory}

Note that in view of the radiation gauge Maxwell's equations (3.3) become

$$
\square A^{\mu}=0,
$$

further, since in the radiation gauge we have $\phi=0$, this becomes

$$
\square \mathbf{A}=0
$$

This is the Klein-Gordon equation for a massless field, and we write

$$
\mathbf{A}(x)=\int \frac{d^{3} \mathbf{k}}{(2 \pi)^{3} 2 k_{0}} \sum_{\lambda=1}^{2} \varepsilon^{(\lambda)}(k)\left[a^{(\lambda)}(\mathbf{k}) e^{-i k \cdot x}+a^{(\lambda) \dagger}(\mathbf{k}) e^{i k . x}\right],
$$

where $\varepsilon^{(\lambda)}(k)$ are known as polarization vectors, $k^{2}=$ $0, k_{0}=|\mathbf{k}|$, and

$$
\mathbf{k} \cdot \varepsilon^{(\lambda)}(k)=0, \quad \varepsilon^{(\lambda)}(k) \cdot \varepsilon^{\left(\lambda^{\prime}\right)}(k)=\delta_{\lambda \lambda^{\prime}} .
$$

The creation and annihilation operators obey the following commutation relations

$$
\begin{gathered}
{\left[a^{(\lambda)}(\mathbf{k}), a^{\left(\lambda^{\prime}\right) \dagger}\left(\mathbf{k}^{\prime}\right)\right]=2 k_{0}(2 \pi)^{3} \delta_{\lambda \lambda^{\prime}} \delta^{3}\left(\mathbf{k}-\mathbf{k}^{\prime}\right),} \\
{\left[a^{(\lambda)}(\mathbf{k}), a^{\left(\lambda^{\prime}\right)}\left(\mathbf{k}^{\prime}\right)\right]=\left[a^{(\lambda) \dagger}(\mathbf{k}), a^{\left(\lambda^{\prime}\right) \dagger}\left(\mathbf{k}^{\prime}\right)\right]=0 .}
\end{gathered}
$$

These commutation relations have the same form as those for scalar field, and have the same interpretation as annihilation and creation operators for photons.

Let us now calculate the vacuum energy. It may be written as

$$
\begin{aligned}
H & =\frac{1}{2} \int d^{3} \mathbf{x}\left(\mathbf{E}^{2}+\mathbf{B}^{2}\right)=\frac{1}{2} \int d^{3} \mathbf{x}\left(\dot{\mathbf{A}}^{2}+(\nabla \times \mathbf{A})^{2}\right) \\
& =\frac{1}{2} \int\left(\dot{\mathbf{A}}^{2}-\mathbf{A} \cdot \nabla^{2} \mathbf{A}\right) d^{3} \mathbf{x} .
\end{aligned}
$$


Substituting (3.8) in (3.11), we obtain after some algebra

$H=\sum_{\lambda=1}^{2} \int \frac{d^{3} \mathbf{k}}{(2 \pi)^{3} 2 k_{0}} \frac{k_{0}}{2}\left[a^{(\lambda)}(\mathbf{k}) a^{(\lambda) \dagger}(\mathbf{k})+a^{(\lambda) \dagger}(\mathbf{k}) a^{(\lambda)}(\mathbf{k})\right]$.

It is clear that the above integral and so the vacuum energy diverges. Unless the "normal ordering" procedure is used.

The propagator for the transverse (physical) photons may be calculated in the same manner as for massive photons, after some algebra we have

$\left\langle 0\left|T\left(A_{\mu}(x) A_{v}(y)\right)\right| 0\right\rangle=i \int \frac{d^{4} k}{(2 \pi)^{4}} \frac{e^{-i k .(x-y)}}{k^{2}+i \varepsilon} \sum_{\lambda=1}^{2} \varepsilon_{\mu}^{(\lambda)}(k) \varepsilon_{v}^{(\lambda)}(k)$.

This propagator bears an infrared divergence in it's imaginary part (Appendix-A).

\subsection{Gupta-Bleuler quantization}

Similar to the previous case, the field operator is defined as follows [2, 4, 11]

$$
\begin{gathered}
\mathbf{A}(x)=\frac{1}{\sqrt{2}}\left[\mathbf{A}^{(p)}(x)+\mathbf{A}^{(n)}(x)\right] \\
\mathbf{A}^{(p)}(x)=\int \frac{d^{3} \mathbf{k}}{(2 \pi)^{3} 2 k_{0}} \sum_{\lambda=1}^{2} \varepsilon^{(\lambda)}(k)\left[a^{(\lambda)}(\mathbf{k}) e^{-i k . x}\right. \\
\left.+a^{(\lambda) \dagger}(\mathbf{k}) e^{i k . x}\right] \\
\mathbf{A}^{(n)}(x)=\int \frac{d^{3} \mathbf{k}}{(2 \pi)^{3} 2 k_{0}} \sum_{\lambda=1}^{2} \varepsilon^{(\lambda)}(k)\left[b^{(\lambda)}(\mathbf{k}) e^{i k . x}\right. \\
\left.+b^{(\lambda) \dagger}(\mathbf{k}) e^{-i k . x}\right]
\end{gathered}
$$

where $\mathbf{A}^{(p)}(x)$ and $\mathbf{A}^{(n)}(x)$ have positive and negative norm respectively, in the sense of Klein-Gordon inner product. $a(\mathbf{k})$ and $b(\mathbf{k})$ are two independent operators. Creation and annihilation operators are constrained to obey the following commutation relations

$$
\begin{gathered}
{\left[a^{(\lambda)}(\mathbf{k}), a^{\left(\lambda^{\prime}\right)}\left(\mathbf{k}^{\prime}\right)\right]=0, \quad\left[a^{(\lambda) \dagger}(\mathbf{k}), a^{\left(\lambda^{\prime}\right) \dagger}\left(\mathbf{k}^{\prime}\right)\right]=0,} \\
{\left[a^{(\lambda)}(\mathbf{k}), a^{\left(\lambda^{\prime}\right) \dagger}\left(\mathbf{k}^{\prime}\right)\right]=2 k_{0}(2 \pi)^{3} \delta_{\lambda \lambda^{\prime}} \delta^{3}\left(\mathbf{k}-\mathbf{k}^{\prime}\right),} \\
{\left[b^{(\lambda)}(\mathbf{k}), b^{\left(\lambda^{\prime}\right)}\left(\mathbf{k}^{\prime}\right)\right]=0, \quad\left[b^{(\lambda) \dagger}(\mathbf{k}), b^{\left(\lambda^{\prime}\right) \dagger}\left(\mathbf{k}^{\prime}\right)\right]=0,} \\
{\left[b^{(\lambda)}(\mathbf{k}), b^{\left(\lambda^{\prime}\right) \dagger}\left(\mathbf{k}^{\prime}\right)\right]=-2 k_{0}(2 \pi)^{3} \delta_{\lambda \lambda^{\prime}} \delta^{3}\left(\mathbf{k}-\mathbf{k}^{\prime}\right),} \\
{\left[a^{(\lambda)}(\mathbf{k}), b^{(\lambda)}\left(\mathbf{k}^{\prime}\right)\right]=0, \quad\left[a^{(\lambda) \dagger}(\mathbf{k}), b^{\left(\lambda^{\prime}\right) \dagger}\left(\mathbf{k}^{\prime}\right)\right]=0,} \\
{\left[a^{(\lambda)}(\mathbf{k}), b^{\left(\lambda^{\prime}\right) \dagger}\left(\mathbf{k}^{\prime}\right)\right]=0, \quad\left[a^{(\lambda) \dagger}(\mathbf{k}), b^{\left(\lambda^{\prime}\right)}\left(\mathbf{k}^{\prime}\right)\right]=0 .}
\end{gathered}
$$

The vacuum is defined by the relations given in Eqs.(2.16)(2.18).

If we calculate the energy operator in terms of the field operator (3.14), we have

$$
\begin{aligned}
H & =\sum_{\lambda=1}^{2} \int \frac{d^{3} \mathbf{k}}{(2 \pi)^{3} 2 k_{0}} \frac{k_{0}}{2}\left[a^{(\lambda) \dagger}(\mathbf{k}) a^{(\lambda)}(\mathbf{k})\right. \\
& \left.+b^{(\lambda) \dagger}(\mathbf{k}) b^{(\lambda)}(\mathbf{k})+a^{(\lambda) \dagger}(\mathbf{k}) b^{(\lambda) \dagger}(\mathbf{k})+a^{(\lambda)}(\mathbf{k}) b^{(\lambda)}(\mathbf{k})\right] .
\end{aligned}
$$

This energy is zero for the vacuum state and it is not needed to use the "normal ordering" procedure. It is also positive for any particles state or physical state $\left|N_{\mathbf{k}}\right\rangle$ (those built from repeated action of the $a^{\dagger}(\mathbf{k})$ 's on the vacuum)

$$
\begin{aligned}
& \left\langle N_{\mathbf{k}}|H| N_{\mathbf{k}}\right\rangle=\sum_{\lambda=1}^{2} \int \frac{d^{3} \mathbf{k}}{(2 \pi)^{3} 2 k_{0}} \frac{k_{0}}{2} \\
& \times\left\langle N_{\mathbf{k}}\left|a^{(\lambda) \dagger}(\mathbf{k}) a^{(\lambda)}(\mathbf{k})\right| N_{\mathbf{k}}\right\rangle \geq 0, \quad\left|0_{\mathbf{k}}\right\rangle \equiv|0\rangle .
\end{aligned}
$$

At this stage we calculate the propagator for the physical photons using Gupta-Bleuler quantization. Using (3.14) in the left hand side of (2.8) we have

$$
\begin{aligned}
\left\langle 0\left|T\left(A_{\mu}(x) A_{v}(y)\right)\right| 0\right\rangle=\frac{1}{2}\left[\left\langle 0\left|T\left(A_{\mu}^{(p)}(x) A_{v}^{(p)}(y)\right)\right| 0\right\rangle\right. & \\
& \left.+\left\langle 0\left|T\left(A_{\mu}^{(n)}(x) A_{\nu}^{(n)}(y)\right)\right| 0\right\rangle\right] \\
& =\frac{1}{2}\left[W_{\mu \nu}^{(p)}(x, y)+W_{\mu \nu}^{(n)}(x, y)\right]
\end{aligned}
$$

By a direct calculation one can show that $W_{\mu \nu}^{(n)}(x, y)=$ $-\left[W_{\mu v}^{(p)}(x, y)\right]^{*}$ and $W_{\mu \nu}^{(p)}(x, y)$ is the two-point function for the positive modes as it is calculated in the previous subsection. Therefore

$$
\begin{array}{r}
\left\langle 0\left|T\left(A_{\mu}(x) A_{v}(y)\right)\right| 0\right\rangle=i I_{m}\left[W_{\mu v}^{(p)}(x, y)\right] \\
=i I_{m}\left[i \int \frac{d^{4} k}{(2 \pi)^{4}} \frac{e^{-i k \cdot(x-y)}}{k^{2}+i \varepsilon} \sum_{\lambda=1}^{2} \varepsilon_{\mu}^{(\lambda)}(k) \varepsilon_{v}^{(\lambda)}(k)\right],
\end{array}
$$

and the propagator for the transverse photons in the Krein space quantization is then

$$
D_{\mu \nu}^{t r}(x-y)=i \mathcal{R} e\left[\int \frac{d^{4} k}{(2 \pi)^{4}} \frac{e^{-i k .(x-y)}}{k^{2}+i \varepsilon} \sum_{\lambda=1}^{2} \varepsilon_{\mu}^{(\lambda)}(k) \varepsilon_{v}^{(\lambda)}(k)\right] \text {, }
$$

which is free of any infrared divergence and it is not need to use the renormalization procedure. Indeed this quantization method acts as an automatic renormalization factor for infrared divergence in the propagators.

\section{CONCLUSION}

Through a generalization of the Gupta-Bleuler quantization introduced in [2], a covariant quantization of the massive and massless vector fields in Minkowski space-time is 
constructed, which is free of any infrared (and ultraviolet) divergences. It has been shown that this method of quantization acts as an automatic renormalization element of the ultraviolet divergence in the stress tensors and a renormalization factor for infrared divergence in the propagators. In other words, using this new quantization method, the ultraviolet divergence in the vacuum energies disappears and the normal ordering is not necessary. Also the two-point functions are automatically renormalized, without any renormalization procedure.

\section{APPENDIX A: SOME USEFUL MATHEMATICAL FORMULA}

In calculating the propagators given in Eqs. (2.9) and (3.13) we encounter the integrals of the form

$$
\begin{gathered}
\int \frac{d^{4} k}{(2 \pi)^{4}} \frac{e^{-i k \cdot(x-y)}}{k^{2}-m^{2}+i \varepsilon}, \\
\int \frac{d^{4} k}{(2 \pi)^{4}} \frac{k_{\mu} k_{v} e^{-i k \cdot(x-y)}}{k^{2}-m^{2}+i \varepsilon}, \\
\int \frac{d^{4} k}{(2 \pi)^{4}} \frac{e^{-i k \cdot(x-y)}}{k^{2}+i \varepsilon},
\end{gathered}
$$

we give a brief explanation about these types of integrations.

Using the following integration formula in $(A .1)$

$$
\int_{0}^{\infty} d s e^{-s\left(k^{2}-m^{2}+i \varepsilon\right)}=\frac{1}{k^{2}-m^{2}+i \varepsilon},
$$

and commuting $d^{4} k$ integration with $d s$ and performing the former, the integral can be solved in terms of the Bessel functions as follows $[11,15]$

$$
\begin{aligned}
& \int \frac{d^{4} k}{(2 \pi)^{4}} \frac{e^{-i k .(x-y)}}{k^{2}-m^{2}+i \varepsilon}=-\frac{1}{8 \pi} \delta(\sigma) \\
& +\frac{m^{2}}{8 \pi} \theta(\sigma) \frac{J_{1}\left(\sqrt{2 m^{2} \sigma}\right)-i N_{1}\left(\sqrt{2 m^{2} \sigma}\right)}{\sqrt{2 m^{2} \sigma}}, \sigma \geq 0,(\mathrm{~A} .5) \\
& \sigma=\frac{1}{2}(x-y)^{2}=\frac{1}{2} g_{\mu \nu}(x-y)^{\mu}(x-y)^{v},
\end{aligned}
$$

where $J_{v}(x)$ and $N_{v}(x)$ are real Bessel functions for real $x$ and v.

Taking twice derivative $\partial_{\mu} \partial_{\nu}$ from both side of Eq. $(A .5)$ we have

$$
\int \frac{d^{4} k}{(2 \pi)^{4}} \frac{k_{\mu} k_{v} e^{-i k \cdot(x-y)}}{k^{2}-m^{2}+i \varepsilon}=-\partial_{\mu} \partial_{\nu} \int \frac{d^{4} k}{(2 \pi)^{4}} \frac{e^{-i k \cdot(x-y)}}{k^{2}-m^{2}+i \varepsilon} .
$$

The limiting values of the various kinds of Bessel functions for small values of their argument are as follows [16]

$$
\begin{aligned}
& J_{v}(x) \rightarrow \frac{1}{\Gamma(v+1)}\left(\frac{x}{2}\right)^{v}, \\
& N_{v}(x) \rightarrow-\frac{\Gamma(v)}{\pi}\left(\frac{2}{x}\right)^{v}, v \neq 0 .
\end{aligned}
$$

In the massless limit, Eq.(A.5) reduces to [15]

$$
\int \frac{d^{4} k}{(2 \pi)^{4}} \frac{e^{-i k \cdot(x-y)}}{k^{2}+i \varepsilon}=\frac{i}{8 \pi^{2} \sigma}-\frac{1}{8 \pi} \delta(\sigma) .
$$

Acknowlegements: I am grateful to referee for useful comments and professor M.V. Takook for his useful discussions.
[1] de Bièvre S., Renaud J., Phys. Rev. D, 57(1998)6230.

[2] Gazeau J-P., Renaud J., Takook M.V., Class. Quantum Grav., 17(2000)1415.

[3] Dehghani M., Rouhani S., Takook M.V. and Tanhayi M.R., Phys. Rev. D, 77(2008)064028.

[4] Takook M.V., Mod. Phys. Lett. A, 16(2001)1691.

[5] de Vega H.J., Ramirez J. and Sanchez N., Phys. Rev. D, 60(1999)044007.

[6] Higuchi A., Kouris S.S., Class. Quantum Grav., 17(2000)3077; 20 (2003) 3005.

[7] Allen B., Phys. Rev. D, 32(1985)3136.

[8] Garidi T., Gazeau J-P, Rouhani S. and Takook M.V., J. Math. Phys., 49(2008)032501.

[9] Gazeau J-P Lett. Math. Phys., 8(1984)507.

[10] Khosravi H., Naseri M., Rouhani S. and Takook M.V., Phys.
Lett. B, 640(2006)48.

[11] Takook M.V., Int. J. Mod. Phys. E, 11(2002)509; 14(2005)219.

[12] Payandeh F., Mehrafarin M. and Takook M.V., Sci. China G, 52(2009)212.

[13] Itxykson C. and Zuber J-B., "Quantum field theory", McGraw-Hill, INC. (1988).

[14] Bordag M., Mohidden U., Mostepanenko V.M., Phys. Rep. 353(2001)1.

[15] Birrel N.D. and Davies P.C.W., "Quantum fields in curved space", Cambridge University press, (1982).

[16] Jackson J.D., "Classical electrodynamics", Third Edition, JOHN WILEY and SONS, INC. (1982). 